[0212-7199 (2004) 21: 12; pp 581-584] ANALES DE MEDICINA INTERNA Copyright (C) 2004 ARAN EDICIONES, S.L.

An. Med. InTERnA (Madrid) Vol. 21, N. ${ }^{\circ} 12$, pp. 581-584, 2004

\section{Niveles inapropiados de digoxina en sangre relativos a 2.849 pacientes procedentes de un hospital universitario: influencia de la edad y el sexo}

\author{
E. PUCHE CAÑAS, I. AOMAR MILLÁN ${ }^{1}$, J. C. CARRILLO SANTOS \\ Unidad de Farmacología Clínica. ${ }^{~}$ Servicio de Patología Médica. Hospital Universitario \\ San Cecilio Granada
}

\author{
INAPPROPRIATE BLOOD LEVELS OF DIGOXIN IN A STUDY OF \\ 2,846 PATIENTS FROM A TEACHING HOSPITAL: THE INFLUENCE \\ OF AGE AND SEX
}

\begin{abstract}
RESUMEN
Objetivos: Detectar el porcentaje de pacientes con niveles inapropiados de digoxina en una amplia muestra y la posible influencia de la edad (mayores o menores de 65 años), y el sexo.

Pacientes y método: Para ello hicimos un estudio retrospectivo sobre las concentraciones de digoxina en sangre de pacientes adultos con insuficiencia cardíaca congestiva y/o fibrilación auricular procedentes del Hospital Universitario San Cecilio entre los años 1992-2002. El análisis estadístico consistió en un análisis descriptivo saeguido de un test de la chi cuadrado para el cálculo de las diferencias entre las distintas variables.

Resultados: Sobre un total de 5.623 determinaciones analíticas y 2.849 pacientes se detectó un 55,4\% de pacientes con niveles de digoxina en sangre inapropiados. Los niveles inapropiados de digoxina fueron significativamente mayores en el grupo de mujeres frente a los varones $(\mathrm{p}<$ $0,001)$. Un $23 \%$ de pacientes tuvieron niveles elevados de digoxina en sangre ( 2 o más $\mathrm{ng} / \mathrm{ml}$ ) y un $16 \%$ con niveles muy bajos $(0,5 \mathrm{ng} / \mathrm{ml} \mathrm{o}$ menos). El promedio de determinaciones analíticas de digoxina en sangre por paciente fue de dos a lo largo del período estudiado.

Conclusiones: Las mujeres y los ancianos tuvieron mayores porcentajes de concentraciones inapropiadas de digoxina en sangre, siendo las mujeres más proclives a tener tasas inapropiadas y elevadas. La monitorización terapéutica de digoxina en sangre no se utiliza todo lo frecuentemente que se debiera, llevando implícito un mal control del paciente digitalizado. Resaltamos el interés de este tipo de estudios.
\end{abstract}

PALABRAS CLAVE: Digoxina. Monitorización terapéutica. Niveles inapropiados. Sexo. Edad.

\section{ABSTRACT}

Objetives: Digoxin is used to treat congestive heart failure and atrial fibrillation. Blood levels need to be monitored to optimize therapeutic performance, detect noncompliance and reduce toxicity. The aim of this study was to evaluate the use of digoxin by measuring blood levels of this drug. The influence of sex and age were also considered.

Patients and method: A retrospective study reviewed determinations of blood digoxin concentration in hospiatalized and ambulatory patients with congestive heart failure, atrial fibrillation, or both, seen at the University of Granada Teaching Hospital (Spain) from 1992 to 2002. A chi square test was applied to results.

Results: A total of 5,623 laboratory tests for digoxin were done for 2,846 adult patients. Patients whose medical record was incomplete were excluded, and the final sample consisted of 2,629 patients. The $55.4 \%$ had inappropriate blood levels of digoxin. Inappropriate concentrations to digoxin were significantly higher in women $(p<0.001)$. The porcentage of patients with high levels of the drug was significantly greater among men $(p<0.001)$. Very low concentrations $(<0.5 \mathrm{ng} / \mathrm{ml})$ were found in $16 \%$ of the patients, with no significant difference between sexes.

Conclusions: We detect a large percentage of older patients with inappropriate levels of digoxin in blood. Women were more likely than men to have high levels to digoxin in blood. There is evidence that therapeutic monitoring of blood levels of digoxin is not done as often as is advisable; this has implications for the care of patients being treated with this drug.

KEY WORDS: Digoxin. Therapeutic monitoring. Inappropriate levels. Gender. Age.

Puche Cañas E, Aomar Millán I, Carrillo Santos JC. Niveles inapropiados de digoxina en sangre relativos a 2.849 pacientes procedentes de un hospital universitario: influencia de la edad y el sexo. An Med Interna (Madrid) 2004; 21: 581-584.

\section{INTRODUCCIÓN}

Datos procedentes de tres ensayos clínicos controlados multicéntricos clásicos como el RADIANCE, PROVED y DIG han demostrado que la digoxina sigue siendo un fármaco de elección en el tratamiento de la insuficiencia cardíaca con- gestiva (ICC), sola o asociada a otros fármacos, al disminuir la morbilidad, mejorar la calidad de vida en los enfermos y reducir las hospitalizaciones por recaídas $(1,2,3)$, y también para control de la fibrilación auricular (FA) (4). El inconveniente mayor que presenta el uso de digoxina es su toxicidad debido al estrecho margen terapéutico que posee siendo nece-

Trabajo aceptado: 3 de septiembre de 2004

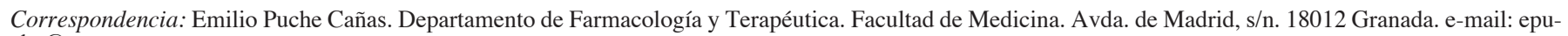
che@.ugr.es 
saria la monitorización terapéutica de sus niveles en sangre. Numerosos estudios han implicado a la digoxina como una causa frecuente de reacciones adversas graves en los enfermos $(5,6)$, en mayor medida entre la población anciana y el sexo femenino $(7,8)$.

Dado que la monitorización de niveles de digoxina en sangre es un dato analítico importante para ajustar la dosis de esta, y que los enfermos presentan con frecuencia niveles inapropiados $(9,10)$, hemos realizado un estudio retrospectivo en una amplia muestra de pacientes procedentes del Hospital Universitario San Cecilio al objeto de obtener información sobre el porcentaje de pacientes que tienen niveles inapropiados de digoxina en sangre, y la participación de la edad y el sexo.

\section{PACIENTES Y MÉTODOS}

Se hizo un estudio retrospectivo entre los años 1992 y 2002 sobre un total de 2.849 pacientes adultos de ambos sexos diagnosticados de una ICC, FA y ICC+FA en tratamiento más de un año con digoxina $0,25 \mathrm{mg} /$ día, con descansos semanales, a los que se les pidió una determinación por diversas causas: control, incumplimiento y toxicidad.

Para la realización del estudio partimos de la base de datos de la Unidad de Farmacología Clínica del Hospital Universitario San Cecilio de Granada, recogiendo la edad, el sexo, las cifras totales de digoxinemia, fecha de la primera digoxinemia, la dosis/día, la causa por la que fue pedido el análisis y la procedencia del enfermo (sala o consulta).

Se catalogó como tasa inapropiada de digoxina en sangre toda aquella cifra fuera del rango terapéutico aceptado, entre 0,8-1,9 ng/ml. La determinación analítica se hizo en una alícuota de suero partiendo de $2 \mathrm{ml}$ de sangre extraída por venopunción en ayunas antes de tomar una nueva dosis de digoxina, excepto para los pacientes procedentes del S. de Urgencia por razones obvias. Fueron usados de forma indistinta dos métodos analíticos automatizados: el TDX Analyzer (Abbott Científica SA) y el MIRA-plus (Dade Behring), ambos con una buena concordancia desde el punto de vista de los resultados analíticos. Las cifras de digoxina en sangre se dividieron en cuatro categorías: normal $(0,8-1,9 \mathrm{ng} / \mathrm{ml})$, alto $(2 \mathrm{ng} / \mathrm{ml} \mathrm{o}$ más), bajo (0,5-0,8 ng/ml), y muy bajo $(0,5 \mathrm{ng} / \mathrm{ml}$ o menos).

Una vez depurados los errores se aplicó el tratamiento estadístico con un análisis descriptivo de la muestra, seguido de un test de la $\chi^{2}$ para hallar las diferencias entre sexo y edad (mayor o menor de 65 años) en base a las cuatro categorias de digoxinemias establecidas. En todos los casos se estableció como límite de significación estadística $\mathrm{p}<0.05$.

\section{RESULTADOS}

Del total de 2.849 pacientes registrados se eliminaron doscientos veinte por tener los datos incompleto, quedando finalmente la muestra en 2.629 enfermos diferentes con una edad promedio de $75 \pm 10$ años (rango de 40-96 años). El 55,4\% de los pacientes presentaron cifras inapropiadas de digoxina (Tabla I), y cuando se estratificaron los pacientes de acuerdo a los cuatro rangos de concentraciones de digoxina en sangre, se observó que un 44,6\% de pacientes estaba en rango terapéutico, un $23 \%$ con cifras elevadas, y un $16 \%$ con cifras muy bajas (Tabla II). Un $12 \%$ de pacientes con cifras de digoxina normales o bajas presentaron signos de toxicidad digitálica, mientras que un $18 \%$ de pacientes con cifras altas no la tuvieron.

TABLA I

DATOS GENERALES DE LA MUESTRA CORRESPONDIENTE AL PERIODO DE ESTUDIO COMPRENDIDO ENTRE LOS AÑOS 1992-2002, AMBOS INCLUSIVE

\begin{tabular}{lcc}
\hline Total pacientes recogidos & 2.849 & \\
Total digoxinemias & 5.623 & \\
Digoxinemias x & 1 cada cinco años & \\
paciente (promedio) & & \\
Pacientes estudiados & 2.629 & \\
Mujeres & 1.498 & $57 \%$ \\
Varones & 1.131 & $43 \%$ \\
Edad (M \pm DS) años & $75 \pm 10$ & \\
Pacientes < 65 años & 263 & $10 \%$ \\
Pacientes > 65 años & 2.359 & $90 \%$ \\
Hospitalizados & 2.129 & $81 \%$ \\
Ambulatorios & 500 & $19 \%$ \\
Con ICC & 947 & $36 \%$ \\
Con FA & 1.078 & $41 \%$ \\
ICC+FA & 604 & $23 \%$ \\
\hline
\end{tabular}

TABLA II

NÚMERO Y PORCENTAJE DE PACIENTES ESTUDIADOS DE ACUERDO A LAS CUATRO CATEGORÍAS ESTABLECIDAS, RESPECTO DE LAS CONCENTRACIONES DE DIGOXINA EN SANGRE (TOTAL 2.629 PACIENTES)

\begin{tabular}{lrc}
\hline Rango & $N^{\circ}$ & $\%$ \\
\hline $0,8-1,9 \mathrm{ng} / \mathrm{ml}$ & 1.173 & 44,6 \\
$0,5-0,8 \mathrm{ng} / \mathrm{ml}$ & 421 & 16,0 \\
$1,9 \mathrm{ng} / \mathrm{ml}$ o más & 610 & 23,2 \\
$0,5 \mathrm{ng} / \mathrm{ml}$ o menos & 425 & 16,1 \\
\hline
\end{tabular}

Cuando se estudió el origen de los enfermos a los que se les pidió una determinación analítica de digoxina hallamos que los mayores porcentajes de pacientes procedieron de Medicina Interna $(47,7 \%)$, del Servicio de Urgencia $(19,5 \%)$, Cardiología (14,2\%), y UVI (8\%), respectivamente. En cuanto a la causa que motivó la petición analítica destacó la sospecha o confirmación de una toxicidad digitálica $(61 \%)$, seguida de un control rutinario $(23 \%)$ y por incumplimiento (16\%).

Cuando analizamos los promedios de digoxinemias $(1,4 \pm$ $1,1$ vs $1,7 \pm 1,3 ; \mathrm{ng} / \mathrm{ml}$; $\mathrm{p}<0,001)$ y las peticiones analíticas de digoxina en sangre ( $57 \%$ vs $43 \%$ ) se obtuvieron diferencias significativas a favor de las mujeres frente a los hombres. En los mayores frente a los menores de sesenta y cinco años ocu- 
rrió igual para la media de digoxinemias $(1,7 \pm 1,1$ vs $1,2 \pm$ $0,9 \mathrm{ng} / \mathrm{ml} \mathrm{p}<0,01)$ y petición de análisis $(90 \%$ vs $10 \%)$. En cuanto a porcentaje de peticiones de análisis destacaron las procedentes de pacientes hospitalizados frente a los ambulatorios ( $81 \%$ vs $19 \%$ ), y un $41 \%$ de peticiones a favor de pacientes con FA (Tabla I).

Cuando se compararon los porcentajes de niveles inapropiados de digoxina entre hombres y mujeres, hallamos una diferencia a favor de las mujeres frente a los varones (29\% vs $15,4 \% ; \chi^{2}=66,18 ; 1$ g.1. $\left.\mathrm{p}<0.001\right)$, y un mayor porcentaje de varones con tasas inapropiadas bajas de digoxina frente a las mujeres $\left(19,2 \%\right.$ vs $13,5 \%, \chi^{2}=16,65 ; 1$ g.l. $\left.p<0,001\right)$. No hallamos diferencias entre sexos respecto de las tasas muy bajas de digoxina en sangre $\left(15,2 \%\right.$ vs $16,8 \%, \chi^{2}=0,81$; 1 g.l. $\mathrm{p}=0,37)$ (Tabla III).

\section{TABLA III}

RELACIÓN DE PACIENTES Y TASAS INAPROPIADAS DE DIGOXINA EN SANGRE SEGÚN EL SEXO (TOTAL 2.629 PACIENTES)

\begin{tabular}{lccccc}
\hline & & \multicolumn{2}{c}{$\begin{array}{c}\text { Varones } \\
(1.131)\end{array}$} & \multicolumn{2}{c}{$\begin{array}{c}\text { Mujeres } \\
(1.498)\end{array}$} \\
\hline Digoxinemia & Total & $n^{\circ}$ & $\%$ & $n^{\circ}$ & $\%$ \\
\hline $1,9 \mathrm{ng} / \mathrm{ml} 0$ más & 610 & 175 & 15,4 & $435^{*}$ & 29 \\
$0,8-0.5 \mathrm{ng} / \mathrm{ml}-1$ & 467 & 241 & 21,3 & $226^{*}$ & 15 \\
$0,5 \mathrm{ng} / \mathrm{ml}-1$ o menos & 425 & 173 & 15,2 & 252 n.s. & 16,8 \\
\hline
\end{tabular}

${ }^{*} p<0,001$, test de la $\chi^{2} ;$ n.s. no significativo.

Cuando tratamos de comparar los porcentajes de enfermos por edades hallamos que el $90 \%$ superó los sesenta y cinco años por lo que no fue posible establecer ningún tipo de comparación fiable entre ellos. En cuanto al porcentaje de pacientes con cifras de digoxina dentro del rango terapéutico hallamos diferencias estadísticamente significativas a favor de los varones frente a las mujeres $\left(56,1 \%\right.$ vs $47,7 \%, \chi^{2}=15 ; 1$ g.1. $\left.\mathrm{p}<0,001\right)$.

No hallamos diferenciación estadísticamente significativas al comparar las digoxinemias de los enfermos con uno o dos descansos semanales en la toma de la digital.

\section{DISCUSIÓN}

Aunque nuestro estudio tiene sus limitaciones, pues sólo se incluyeron aquellos pacientes a los que se les pidió una determinación de digoxina en sangre en el tiempo que abarcó el estudio. Sin embargo, y es lo importante a nuestro juicio, más de la mitad de los pacientes presentaron cifras inapropiadas de digoxina, por exceso o por defecto, y un $23 \%$ tuvieron niveles altos en el momento de realizar el análisis. El elevado porcentaje de pacientes con cifras altas de digoxina en sangre es un dato importante que requiere confirmación dada la relación descrita recientemente entre muerte e incremento de las cifras de digoxina por encima de $1,5 \mathrm{ng} / \mathrm{ml}$, sobre todo en mujeres $(8,11)$, que en nuestra revisión fueron un grupo mayoritario.

En cuanto a los niveles inapropiados de digoxina inferiores a $0,8 \mathrm{ng} / \mathrm{ml}$, hallamos un $32 \%$ de pacientes en esta situación lo que se podría interpretar como la consecuencia directa de una dosificación incorrecta o de un incumplimiento por parte del paciente que conlleva una situación de ineficacia terapéutica (12). Ultimamente se han descrito efectos beneficiosos para los niveles bajos de digoxina en sangre, para algunos autores llega hasta $0,5 \mathrm{ng} / \mathrm{ml}$ (13), por lo que el rango terapéutico aceptado está en discusión en la actualidad.

Si las cifras altas de digoxina en sangre se asocian con un incremento de la toxicidad y de muerte en los enfermos, las dosis muy bajas $(0,5 \mathrm{ng} / \mathrm{ml}$ o menos $)$ se asocian con una ineficacia terapéutica, y son el resultado de un franco incumplimiento o de una infradosificación de la digital, que llegado el caso, y si el cuadro clínico lo permite, podría plantearse la supresión de la misma. En nuestro estudio hallamos un $16 \%$ de pacientes en esta situación.

Por otro lado, hemos detectado que las mujeres de nuestra revisión presentaron niveles inapropiados de digoxina en sangre superiores, así como de concentraciones medias de digoxina en sangre, respecto del varón. Diversos autores describen una mayor proporción de digoxinemias elevadas en mujeres respecto de los hombres $(10,14)$ por causa de las diferencias farmacocinéticas existentes entre ambos sexos; la mujer tiene un aclaramiento renal y una masa corporal menores respecto del varón, dos parámetros importantes en la cinética de la digoxina $(14,15)$.

Respecto de la edad, no fue posible comparar las categorías de digoxina en los pacientes ya que el $90 \%$ de todos ellos fueron mayores de sesenta y cinco años. Al ser la ICC y la FA enfermedades muy prevalentes entre los ancianos $(16,17)$ los tratamientos con digoxina se localizan mayoritariamente entre ellos, y de igual forma los pacientes con niveles inapropiados de digoxina . La vejez es un factor de riesgo añadido al tratamiento con digoxina por las mermas funcionales propias de su estado y la comorbilidad añadida $(18,19)$, por eso se requieren controles de este fármaco en sangre más rutinarios, si cabe, entre ancianos digitalizados.

De otra parte, nuestros resultados sugieren la existencia de una baja petición de digoxinemia, entre los pacientes estudiados, basándonos en el hallazgo de una determinación por paciente cada cinco años de promedio, y siendo la sospecha de intoxicación la causa más frecuente a la hora de pedirla. Este es un dato interesante que también requiere confirmación porque en este tipo de enfermos se ha descrito un elevado incumplimiento para la digoxina (20) y un incremento de la morbi-mortalidad derivada del propio incumplimiento (21). La monitorización periódica de las cifras de digoxina en sangre reduce los casos de intoxicación digitálica y mejora su eficacia terapéutica $(22,23)$.

En último lugar comentar que no todos los pacientes que tuvieron cifras altas de digoxina en sangre presentaron signos de toxicidad, y que pacientes con cifras consideradas normales o bajas tuvieron signos de toxicidad digitálica. Esta discordancia se ha descrito en la literatura $(24,25)$, debiéndose tanto a la presencia de sustancias digoxin-similares que pueden interferir analíticamente aumentando falsamente la digoxinemia, (26), como a una sensibilidad mayor del miocardio a la digoxina por interacciones con otros fármacos o por disturbios electrolíticos, hipopotasemia e hipomagnesemia (24), en los casos de cifras bajas de digoxina que se acompañan de signos de toxicidad.

Concluimos llamando la atención sobre el elevado porcentaje de pacientes con niveles inapropiados de digoxina en sangre. La digoxina es un fármaco útil y muy usado en el tratamiento de la ICC y de la FA cuya monitorización terapéutica aporta una valiosa información al médico a la hora de ajustar 
la dosis, diagnosticar la toxicidad o valorar el cumplimiento del enfermo (17,23). La petición de sus niveles sanguíneos de forma rutinaria ayuda a mejorar la calidad de la prescripción médica, aumenta su rendimiento terapéutico y reduce su toxicidad sobre todo en mujeres y ancianos.

\section{AGRADECIMIENTOS}

Al Prof. Rico Irles por sus acertados comentarios y observaciones al trabajo. A la Sta. Cruz Muñoz y al Dr. Saucedo por su ayuda en el manejo de la base de datos.

\section{Bibliografía}

1. Parker M, Gheorghiade M, Young JB, et al. RADIANCE Study. Withdrawal of digoxin from patients with chronic heart failure treated with angiotensin-converting-enzyme inhibitors. N Engl J Med 1993; 329: 1-7.

2. Uretsky BF, Young JB, Shahidi FE, et al. PROVED Investigative Group. J Am Coll Cardiol 1993; 22: 955-62.

3. The effect of digoxin on mortality and morbility in patients with heart failure. The Digitalis Investigation Group (DIG). N Engl J Med 1997; 336: 525-33.

4. Fuster V, Ryden L. ACC/AHA/ESC Guidelines for the management of patients with atrial fibrillation. J Am Col Cardiol 2001; 38: 1231-65.

5. Marik P, Fromm L. A case series of hospitalized patients with elevated digoxin levels. Am J Med 1998; 105: 110-15.

6. McDonell P, Jacobs M. Hospital admissions resulting from preventable adverse drug reactions. Ann Pharmacother 2002; 36: 1331-6.

7. Pahor M, Guralnik JM, Gambassi G, et al. The impact of age on risk of adverse drug reactions to digoxin. J Clin Epidemiol 1993; 46: 1305-14.

8. Rathore S, Wang Y, Krumholz H. Sex-based differences in the effect of digoxin for the treatment of heart failure. N Engl J Med 2002; 347: 1403-11.

9. Piergegies A, Worwag E, Atkinson A. A concurrent audit of high digoxin plasma levels. Clin Pharmacol Ther 1994; 55: 353-58.

10. Cañas F, Milenko J, Tanasijevic M, Maluf N, Bates D. Evaluation the appropriateness of digoxin level monitoring. Arch Intern Med 1999; 159: 363-68.

11. Rathore S, Curtis J, Wang Y, Bristow M, Krumholz H. Association of serum digoxin concentration and outcomes in patients with heart failure. JAMA 2003; 289: 871-78.

12. Carbonin $\mathrm{P}$, Zuccalá G. Optimal digoxin concentrations for patients with heart failure. JAMA 2003; 289: 2643.

13. Young J, Gheorghiade M, Packer M, Uretsky B, Hull H. Are low serum levels of digoxin effective in chronic heart failure? Evidence challenging the accepted guidelines for a therapeutic serum level of the drug. J Am Coll Cardiol 1993; 21: 378.

14 Meibolhm B, Beierle I, Derendorf H. How important are gender differences in pharmacokinetics? Clin Pharmacokinet 2002; 41: 329-42.
15. Mooradian AD. Digitalis: An update to clinical pharmacokinetics, therapeutic monitoring techniques and treatment recommendations. Clin Pharmacokinetics 1988; 15: 165-78.

16. Sutton GC. Epidemiologic aspects of heart failure. Am Heart J 1990; 120: $1538-40$.

17. Mittelmark MB. Prevalence of the cardiovascular diseases among older adults: The Cardiovascular Health Study. Am J Epidemiol 1993; 137: 311-17.

18. Rich M, McSherry F, Williford W, Salim Y. Effect of age on mortality, hospitalitations and response to digoxin in patients with heart failure: The DIG Study. JACC 2001; 38: 806-13.

19. Miura T, Kojima R, Mizutani M, Shiga Y, Takatsu F, Suzuki Y. Effect of digoxin noncompliance on hospitalization and mortality in patients with heart failure in long.term therapy: a prospective cohort study. Eur J Clin Pharmacol 2001; 57: 77-83.

20. Cuena Boy R, Ortiz de Apodaca MA. Incumplimiento del tratamiento terapéuticoambulatorio en ancianos tratados con digoxina. Aten Prim 1998; 21: 302-6.

21. Litaker J, Chou J. Patterns of pharmacologic treatment of congestive heart failure in elderly nursing home residents and related issues. A review of the literature. Clin Ther 2003; 25: 1918-35.

22. Dhume DW, Greenblatt DJ, Koch-Weser J. Reduction of digoxin toxicity associated with measurement of serum levels. Ann Intern Med 1974; 80: 516-19.

23. Wing D, Duff HJ. Impact of a therapeutic drug monitoring program for digoxin. Arch Intern Med 1987; 147: 1405-8.

24. Mitchell G. Adverse reactions to digoxin in tour patients with normal or low serum digoxin levels. Med J Aust 1997; 167: 111.

25. Maíquez P, Abadín JA, Jiménez P, Sánchez A, Durán JA. Intoxicaciones digitálicas sospechadas y confirmadas. An Med Interna (Madrid) 2003; 20: 399-402.

26. Dasgupta A. Endogenous and exogenous digoxin-like inmunoreactive substances. Impact on therapeutic drug monitoring of digoxin. Am J Clin Pathol 2002; 118: 132-40. 